\title{
Mauriac Syndrome - a rare type 1 diabetes mellitus complication and an opportunity for intervention
}

Pedro Souteiro', Sandra Belo ${ }^{1,2,3}$, Maria Manuel Costa ${ }^{1,2,3}$, Fátima Carneiro ${ }^{4}$, Davide Carvalho ${ }^{1,2,3}$

${ }^{1}$ Department of Endocrinology, Diabetes and Metabolism, Centro Hospitalar São João, Porto, Portugal, ${ }^{2}$ Faculty of Medicine of University of Porto, Porto, Portugal, ${ }^{3}$ Instituto de Investigação e Inovação em Saúde, University of Porto, Porto, Portugal, ${ }^{4}$ Department of Pathology, Centro Hospitalar São João, Porto

\section{INTRODUCTION}

Mauriac Syndrome is characterized by the presence of hepatomegaly, growth retardation, delayed puberty and cushingoid features. This entity is traditionally diagnosed in patients with type 1 diabetes mellitus (T1DM) and poor glycaemic control. However, the impact of metabolic control in the normalization of hepatic analytic profile is not clarified.

\section{METHODS}

Retrospective study of five patients with T1DM with hepatic histopathology indicative of Mauriac Syndrome.

\section{RESULTS}

\begin{tabular}{|c|c|c|c|c|c|c|c|c|}
\hline Patient & Sex & Age & $\begin{array}{l}\text { End organ } \\
\text { damage } \\
\end{array}$ & $\begin{array}{l}\text { Hb/1 } 1 \mathrm{c} \\
(\%) \\
\end{array}$ & $\begin{array}{c}\text { AST } \\
(10-37 \mathrm{U} / \mathrm{L}) \\
\end{array}$ & $\begin{array}{c}\text { ALT } \\
(10-37 \mathrm{U} / \mathrm{L}) \\
\end{array}$ & $\begin{array}{l}\text { Hepatomegaly* } \\
(<15 \mathrm{~cm})\end{array}$ & $\begin{array}{l}\text { Other Mauriac } \\
\text { Syndrome features }\end{array}$ \\
\hline 1 & M & 22 & $\begin{array}{l}\text { Retinopathy } \\
\text { Nephropathy }\end{array}$ & 15,7 & 226 & 176 & $17 \mathrm{~cm}$ & $\begin{array}{l}\text { - Cushingoid features } \\
\text { - Short stature }-160 \mathrm{~cm}^{\#}\end{array}$ \\
\hline 2 & $\mathrm{~F}$ & 29 & Retinopathy & 10,9 & 991 & 461 & $25 \mathrm{~cm}$ & \\
\hline 3 & $\mathrm{~F}$ & 20 & $\begin{array}{l}\text { Retinopathy } \\
\text { Nephropathy } \\
\text { Neuropathy }\end{array}$ & 14,0 & 102 & 39 & $22 \mathrm{~cm}$ & - Short stature $-156 \mathrm{~cm}^{+}$ \\
\hline 4 & $\mathrm{~F}$ & 20 & $\begin{array}{l}\text { Retinopathy } \\
\text { Nephropathy } \\
\text { Neuropathy }\end{array}$ & 11,7 & 227 & 439 & $23 \mathrm{~cm}$ & $\begin{array}{l}\text { - Cushingoid features } \\
\text { - Short stature }-155 \mathrm{~cm}^{+}\end{array}$ \\
\hline 5 & $\mathrm{~F}$ & 15 & Retinopathy & 11,7 & 150 & 102 & $21 \mathrm{~cm}$ & - Short stature $-154 \mathrm{~cm}$ \\
\hline
\end{tabular}

Table 1. Baseline characteristics of the study population.

*medioclavicular measures on ecography; \# under the 3rd percentile on WHO growth charts; + under the 10th percentile on WHO growth charts

All 5 patients underwent liver biopsy to clarify the alterations on the enzymes of hepatic cytolysis
Histopathological features of Mauriac Syndrome: Nuclear glycogenization Cytoplasmatic ballooning
- Insulin therapy intensification

Therapy education reinforcement

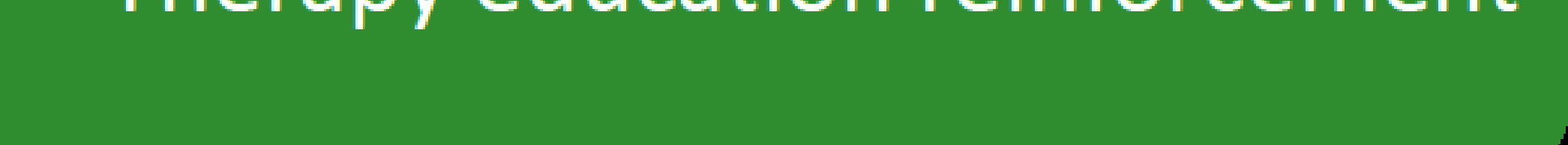

\section{HbA1c evolution after the diagnosis}

17

15

13

11

9

7

-Patient 1 -Patient $2-$ Patient 3 -Patient $4-$ Patient 5

\section{0}

400

300

0
ALT levels evolution after the diagnosis

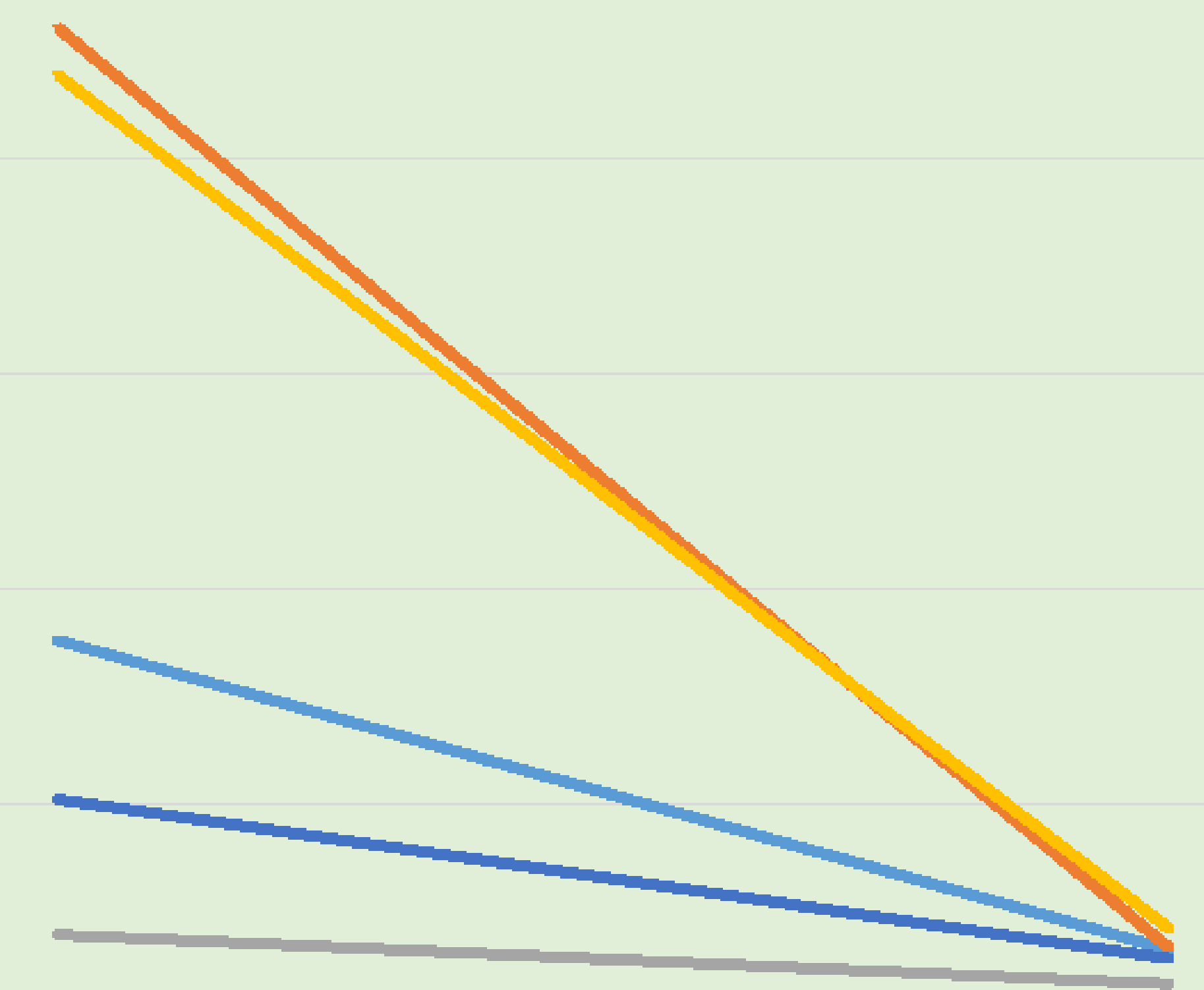

-Patient 1 -Patient 2 -Patient 3 -Patient 4 12Matient 5

\section{CONCUSIONS}

Mauriac Syndrome is rare and not readily diagnosed by most clinicians. The improvement of metabolic control seems to lead to hepatic enzymes normalization in these patients, reinforcing the importance of early intervention.

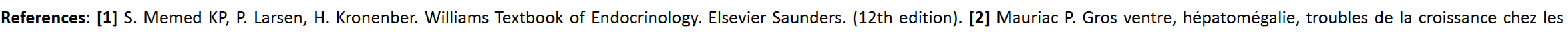
enfants diabétiques, traités depuis plusieurs années par l'insuline. Gas Hebd de Soc Med de Bordeaux. 1930(26):page 402. 